\title{
Restarting Anticoagulants after a Gastrointestinal Hemorrhage- Between Rockall and a Hard Place
}

\author{
Sachin J Shah, MD, MPH'*; Mark H Eckman, MD²
}

'Division of Hospital Medicine, University of California, San Francisco, California; ${ }^{2}$ Division of General Internal Medicine, University of Cincinnati College of Medicine, Cincinnati, Ohio.

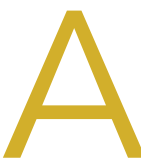

nticoagulant use to prevent ischemic strokes in patients with atrial fibrillation (AF) continues to be one of the most challenging decisions facing patients and their physicians, in large part due to significant patient-to-patient variation in both AF-related stroke risk and anticoagulant-associated hemorrhage risk. Now, add a layer of complexity-.how should one approach anticoagulant use following an adverse event such as an acute upper gastrointestinal (GI) hemorrhage? On the one side, the risk of ischemic stroke, and on the other, the risk of recurrent bleeding, either of which can lead to death or disability. Making this decision requires humility, clinical acumen, shared decision-making, and data.

Data on this subject are sparse.1,2 Observational studies show that patients who restart anticoagulants after Gl hemorrhage experience fewer ischemic strokes. These studies also show that patients who restart anticoagulant therapy are healthier than those who do not-in measurable ways and, importantly, in unmeasurable ways. Thus far, observational studies have not sufficiently dealt with confounding by indication; that is, patients who restart anticoagulants are fundamentally different than patients who do not.

In this issue of the Journal of Hospital Medicine ${ }^{\circledR}$, Pappas et al. focus on the optimal timing of resuming oral anticoagulation in patients who have sustained acute upper GI bleeds while receiving oral anticoagulation for $\mathrm{AF}^{3}{ }^{3}$ They use a microsimulation modeling approach to address this question, by creating a synthetic population of patients reflective of age, gender, and comorbidities in a United States population of patients with AF. Using data from epidemiologic studies that describe the risk of rebleeding, hemorrhagic complications, and ischemic stroke as well as the quality of life associated with each of these events, the authors have constructed a decision analytic model to determine the optimal day to restart anticoagulation. This modeling approach mitigates confounding by indication, a limitation of observational studies. They report that the optimal day to restart anticoagulant therapy is in the range of 32-51 days. As one would predict, when using direct-acting anticoagulants and for patients with high stroke risk, the investigators find that restarting therapy earlier is associated with greater benefit. These findings help to untangle

*Corresponding Author: Sachin J Shah, MD, MPH; E-mail: sachin.shah@ucsf. edu; Telephone: (415) 862-8616; Twitter: @sachinjshah.

Received: March 13, 2019; Revised: March 22, 2019; Accepted: April 2, 2019

๑ 2019 Society of Hospital Medicine DOI 10.12788/jhm.3221 a knot of risk and benefits facing patients with AF following an acute GI hemorrhage.

Interpreting the results relies on an understanding of the strengths and weaknesses of simulation modeling and the data used in the analysis. Like any research method, the devil is in the details. Stitching together event rates and outcomes from multiple studies, the results of a simulation model are only as good as the studies the model draws from. In particular, assumptions regarding the time-dependent decline in rebleeding risk are a critical component of determining the optimal time to resume anticoagulation. The authors had to make multiple assumptions to project the 24-hour risk of rebleeding determined from the Rockall score to estimate the risk of rebleeding over the next days to months. ${ }^{4}$ Consequently, the results are likely overly precise. Practically, 30-50 days or four to eight weeks may better reflect the precision of the study findings.

Results on optimal timing of resuming anticoagulation therapy are most applicable for patients when the decision to restart anticoagulants has already been made. We part ways with the authors in their conclusion that these results confirm that anticoagulants should be restarted. There are multiple appropriate reasons why anticoagulant therapy should not be restarted following an acute upper GI hemorrhage. For example, in observational studies, patients not restarted on anticoagulant therapy were more likely to have a history of falls and to have had severe bleeds. ${ }^{1}$ Furthermore, patients who do not restart therapy are more likely to die in follow-up. It is tempting to use this fact to support restarting anticoagulants. However, when the causes of death are examined, the vast majority of deaths were unrelated to thrombosis or hemorrhage. ${ }^{2}$ Patients with AF are older and have multiple comorbidities and life-limiting conditions. Accordingly, the results of this study are better used to engage patients in shared decision-making and contextualized in the broader picture of patients' health and goals. ${ }^{5}$

Restarting anticoagulants after a GI hemorrhage is a difficult and high-stakes clinical decision. The study by Pappas et al. uses a simulation model to advance our understanding about the optimal timing to restart anticoagulants. By integrating the dynamic risk of ischemic stroke and recurrent hemorrhage following Gl hemorrhage, they estimate the maximal benefit when anticoagulants are restarted between 30 days and 50 days after hemorrhage. The results of their analysis are best used to inform timing among patients where the decision to restart anticoagulants has already been made. The analysis also provides a useful starting point for shared decision-making by highlighting that the optimal net benefit is influenced by 
patient-to-patient variation in the underlying AF-related stroke risk and anticoagulant-associated rebleeding risk.

Disclosures: Dr. Shah has nothing to disclose. Dr. Eckman reports grants from Heart Rhythm Society/Boehringer-Ingelheim and grants from Bristol-Myers Squibb/Pfizer Education Consortium, outside the submitted work.

\section{References}

1. Qureshi W, Mittal C, Patsias I, et al. Restarting anticoagulation and outcomes after major gastrointestinal bleeding in atrial fibrillation. Am J Cardiol. 2014;113(4):662-668. doi: 10.1016/j.amjcard.2013.10.044
2. Witt DM, Delate T, Garcia DA, et al. Risk of thromboembolism, recurrent hemorrhage, and death after warfarin therapy interruption for gastrointestinal tract bleeding. Arch Intern Med. 2012;172(19):1484-1491. doi: 10.1001/ archinternmed.2012.4261.

3. Pappas MA, Evans N, Rizk MK, Rothberg MB. Resuming anticoagulation following upper gastrointestinal bleeding among patients with nonvalvular atrial fibrillation-a microsimulation analysis. J Hosp Med. 2019;14(7):394-400. doi: 10.12788/jhm.3189.

4. Rockall TA, Logan RF, Devlin HB, Northfield TC. Risk assessment after acute upper gastrointestinal haemorrhage. Gut. 1996;38(3):316-321. doi: 10.1136/ gut.38.3.316.

5. Tinetti ME, Naik AD, Dodson JA. Moving from disease-centered to patient goals-directed care for patients with multiple chronic conditions: patient value-based care. JAMA Cardiol. 2016;1(1):9-10. doi: 10.1001/jamacardio.2015.0248. 